\title{
LA INTELIGENCIA ARTIFICIAL COMO GATO DE SCHRÖDINGER EN EL ARTE: ¿OBJETO Y SUJETO DE DERECHO?
}

\author{
Jorge Villalobos Portales \\ Universidad de Málaga
}

\begin{abstract}
Resumen: Este artículo analiza la situación jurídico-filosófica de la Inteligencia Artificial y el robot inteligente sobre ser un sujeto de Derecho para ser considerado persona creativa o autor. Se analiza la contradicción que supone permitir que un objeto pueda presentar la dualidad jurídica de ser protegido como objeto que es y a la vez considerársele sujeto por la obra resultante, un menoscabo o vulgarización para el sujeto de Derecho como sujeto moral al plantear la fictio legis de un objeto moral, lo cual no es concebible. Se propone, en cambio, una alternativa a tal aporía, más ficticia que real.
\end{abstract}

Palabras clave: Inteligencia Artificial; sujeto de Derecho; originalidad; cuasi autor; personalidad ciberhumanoide.

Artificial Intelligence as Schrödinger's cat in art: object and subject of Law?

Abstract: This article analyzes the legal-philosophical situation of Artificial Intelligence and the intelligent robot on being a subject of law to be considered a creative person or author. The contradiction involved in allowing an object to present the legal duality of being protected as an object that is and at the same time considered subject by the resulting work is analyzed, an impairment or vulgarization for the subject of Law as a moral subject when proposing the fictio legis of a moral object, which is not conceivable. Instead, an alternative to such an aporia is proposed, more fictitious than real.

Keywords: Artificial Intelligence; subject of law; originality; quasi-author; cyberhumanoid personality.

Recibido: 24 de mayo de 2021

Aceptado: 6 de octubre de 2021

DOI 10.24310/NATyLIB.2021.vi15.12773 


\section{Introducción: una ficción más real que ficticia}

Dentro del ámbito de la Filosofía del Derecho, pocas cuestiones suscitan tanta atracción como inquietud semejable a los dilemas en torno a la Inteligencia Artificial (IA). En una actualidad tan virtualizada como por momentos casi distópica donde, por ejemplo, un Tribunal de Shenzen, de la provincia de Guandong, reconocía en enero de 2020 copyright a la IA Dreamwriter (Chávez Valdivia, 2020) o el Tribunal de la Haya de Primera instancia resolvía en febrero de ese mismo año sobre la aplicación con sesgos discriminatorios de la IA SyRI en los procesos resolutivos y deliberativos que llevaba a cabo para el gobierno de los Países Bajos (Bendito Cañizares, 2021). Otra IA como es la denominada Prometea (Corvalán, 2017) en Argentina también resuelve sobre ámbitos de la administración pública. $\mathrm{O}$ el ejemplo de Estados Unidos, que se detectó que estaba discriminando con sesgos racistas en función de parámetros como fue el del domicilio. En Dubai se realizan carreras de camellos con jinetes robots. La firma norteamericana Watson "fichó" a la IA jurista Ross para su plantilla como jefe de cincuenta abogados. La robot Sophia tiene asignada nacionalidad y sexo. Mitihido Matsuda se presentó a las elecciones de 2017 en el distrito de Tama, Tokio, y fue tercera fuerza política. En un concurso nacional de Japón, entre las 1450 obras presentadas, 11 eran escritas por una IA y entre los pocos finalistas se encontraba una obra de ellas.

En el ámbito de la creación, los casos conocidos como el de The next Rembrandt (Yanisky-Ravid, 2017), que consiguió crear un cuadro nuevo del autor, el caso de AARON o el de Flowmachine y la canción generada por IA Daddy's car (Díaz-Limón, 2016) dentro de la música. Incluso en el ámbito como puede ser el de la Propiedad Industrial, se topó con distintas situaciones, la más llamativa, la del caso Dabus (Azuaje Pirela \& Finol González, 2020), donde el inventor quiso patentar dos creaciones asignando como inventor, a la IA pues era, según él, quien había producido tales invenciones y que él nada tuvo que intervenir en el proceso. Robot influencers creando contenido en las distintas plataformas de red social junto a otros influencers (de carne y hueso). La robot Roxxy dentro del mundo del trabajo sexual. La aplicación de IA y realidad virtual para reencontrar a una madre con su hija fallecida a través del experimento I met you o la aplicación terapéutica (Marcos, 2015). Estos pocos 
casos comentados se encuentran entre los más recientes, donde la lista continúa.

Lo que reflejan es que aquellos escenarios normalmente transitados más por la realidad literaria que la cotidiana se han ido construyendo a un ritmo superior en cuanto a sus posibles resoluciones filosóficas y jurídicas, pues, entre los conflictos esenciales está la cuestión de ante qué estamos, si un objeto de Derecho cuyos fuegos de artificio nos abruman igual que encandilan los desvaríos más futuribles que futuros o, en cambio, la evolución del objeto a sujeto de Derecho es una incógnita alquímica resuelta gracias a las nuevas tecnologías como tantos otros interrogantes dilucidados por el desarrollo. O quizá como el experimento teórico de Schrödinger, donde se encuentra un gato encerrado en una caja con un cuenco de veneno, el gato está vivo y muerto al mismo tiempo hasta que se destape la incertidumbre. ¿La IA y, en su mayor proyección "antropomórfica", los robots inteligentes, son objeto y sujeto de Derecho dependiendo de qué tipo en concreto se trate y bajo determinados preceptos? ¿Puede ser una IA una persona electrónica no solo dentro del ámbito del Derecho, sino fuera de él, y un robot inteligente un ciudadano tal y como se comprende a una persona física, humana? O, por el contrario, ¿no es quizá la formulación de estas analogías un brochazo intelectual reduccionista de lo que supone ser persona? ¿No estamos exigiendo unas expectativas demasiado irrealizables a lo que no deja de ser un bien mueble de gran complejidad?

El presente trabajo analizará tales preguntas en las cuestiones, primero, sobre la pertinencia o no de considerar y en qué tipos en concreto a una IA o un robot inteligente como sujetos de Derecho para proponer una alternativa sobre una propuesta intermedia entre objeto y sujeto donde se pueda separar y discernir dentro del término sujeto de Derecho el sujeto moral y sujeto económico como ente que interacciona en la sociedad y produce responsabilidades exigibles a terceros y cierto grado de ostentación de derechos en el ámbito económico que puede existir en un plano formal (pues la ingeniería jurídica y la fictio legis todo lo puede sobre un papel) sin confundir que este proceso es un artificio filosófico-jurídico para ser un instrumento vehicular facilitador. Al igual que se puede discernir que una Termomix no es lo mismo que la IA Dabus, también se debe discernir que un robot inteligente no es una persona 
como lo es un ser humano, pues tampoco lo es siquiera la persona jurídica (un sujeto económico compuesto por los sujetos, estos sí, morales que son los seres humanos). En segundo lugar, se tratará estas cuestiones en la disciplina donde más problemática surge, la creación y el reconocimiento de categorías como autor en la Propiedad Intelectual. Por último, concluir sobre la aporía jurídica en que consiste plantear a la IA como sujeto, por muy específicos casos que atañen, pues es aceptar un ente cuya naturaleza es de objeto con una cualidad cuyo telos la posicione como sujeto exhortaría a aceptar autoría a un objeto, un objeto-autor, un objeto-sujeto.

\section{Qué inteligencia artificial puede plantear este tipo de problemática}

En primer lugar, es preciso diferenciar que, dentro del amplísimo mundo de la IA, solo un determinado tipo concreto es el cual puede suscitar estos problemas, pues el resto ni siquiera entran en tal cuestión ni escapan de ser otro mero objeto más del Derecho y de la realidad social. Es preciso diferenciar que existen, a grandes rasgos, tipos de IA débil y de tipo fuerte. Sobre estas cualidades, Grandhi las recoge en una diferenciación de distintos niveles en dos bloques. Primero, entender que están las IA de tipo débil y la de tipo fuerte por un lado y, después, las de aprendizaje simbólico y no simbólico (Grandhi, 2020).

Dentro del primer bloque, en el caso de las IAs de tipo débil, su principal característica es la de realizar tareas específicas, de no ser reactiva, lo que significa que no actúa de manera autónoma, no es flexible, y por tanto depende de la programación humana a través de la imitación, pues no consta de ninguna capacidad de raciocinio propia ni tampoco de aprendizaje. En el segundo caso, las de tipo fuerte, sí tiene la capacidad de llevar a cabo tareas que suelen ser abarcadas por el intelecto humano. Esto le permite una flexibilidad de actuación para la resolución de problemas, donde aquí sí participa de una manera proactiva, con cualidades de autoprogramación a través de técnicas como es la retroalimentación mediante redes neuronales complejas.

Luego, que en ese nivel de programación se encuentran algoritmos supervisados y no supervisados; además, los procesos pueden ser de carácter simbólico, donde se encuentran disciplinas desde el machine learning, el Deep 
learning, redes neuronales, autoaprendizaje. Dentro de las de tipo simbólico y no simbólico, las del primer grupo, se centran sobre todo en los ámbitos de la lógica matemática, que a su vez se inspira en sistemas de reglas, así como representaciones de conocimiento. En cambio, a diferencia de este tipo, las de no simbólico se centran en sistemas más semejables a simulaciones o emulaciones del comportamiento humano, como puede ser el proceso en que funciona el sistema nervioso, como es el sistema de redes neuronales, o el autoaprendizaje que se da en los organismos vivos. Entre estos dos grupos, es el segundo el que encarna la rama más "ambiciosa" en cuanto que busca simular o asemejarse, al menos, a los distintos sistemas y procesos que operan en el cerebro. En pocas palabras, la IA que puede suscitar estos dilemas es solo la de tipo fuerte, no supervisado (Amado Osorio, 2020), de carácter no simbólico y, además, con una peculiaridad mayor, el proceso resolutivo entre el input y el output de información donde la evolución de la IA se escapa al control y la supervisión de cualquier tercero (Azuaje Pirela \& Finol González, 2020), como el propio programador, esto es, el concepto de "caja negra":

En esencia, esto significa que los algoritmos no pueden ofrecer una explicación detallada acerca de cómo llegan a un determinado resultado. Es decir, no puede establecerse cómo el sistema de IA evalúa y pondera los datos y la información que procesa. Por eso se habla de "cajas negras". Se llama sistema de caja negra a la herramienta computacional en la que uno entiende los datos ingresados y los resultados, pero no comprende el procedimiento subyacente. Aquí el código es inescrutable, porque el programa "evoluciona" y los seres humanos no pueden entender el proceso que siguió la programación para lograr una solución determinada. ${ }^{1}$

La principal característica reside en la ausencia del factor humano en los procesos que lleve a cabo la IA, lo cual implica que la posible definición de "autonomía" que se plantea es en una concepción negativa del término: entendemos que una IA puede ser autónoma o independiente siempre que ningún 
humano pueda intervenir o supervisar el proceso desarrollado por la IA, a diferencia de la autonomía del individuo que es desde una concepción positiva, donde emana de sí mismo su autonomía. Otra característica es que todos los procesos descritos consisten en la emulación y la simulación del proceso humano, es decir, la IA solo puede abarcar el campo técnico incluso en el factor volitivo del ser humano, sólo podrá copiar, simular, emular la capacidad decisoria, el corpus mechanicum del ser humano, sin embargo, el animus vivendi tras el factor técnico y el cual supone el origen del incentivo indisoluble de la persona se escapa a la IA ya que la traducción intelectual de las cualidades supra técnica de tomar una decisión o no, de crear o no una obra están fuera del alcance de toda programación. Que pueda emular al ser humano no significa que sea humano.

\section{De objeto a sujeto: una alquimia axiológica de la personalidad jurídica}

María José Santos González, coordinadora del Departamento Jurídico del Instituto Nacional de Ciberseguridad de España, comentaba en el estudio "Regulación legal de la robótica y la inteligencia artificial: retos de futuro",este tipo de cuestiones sobre estudios tanto de la consultora Gartner donde, en teoría, en 2020 conversaríamos más con robots o IAs que con personas, como nuestras propias parejas, por ejemplo. También, alertaba sobre la preocupación de varios psicólogos en que esto supondría difuminar la a veces aparentemente ya escasa línea entre realidad y ficción, lo natural frente a lo artificial. Sin embargo ¿se puede equiparar la mente humana con la artificial?, ¿no nos obcecamos en el corpus mechanicum en vez del corpus mysticum oculto tras la fachada de la técnica? Tal y como se pregunta Santos González, "¿pueden cometer delitos? ¿Debe ser un robot juzgado por sus actos igual que una persona y a su mismo nivel por ser inteligente artificialmente, aunque carezca de emociones y sentimientos reales? ¿Cómo podemos controlar al robot?" (Santos González, 2017).

O, por el contrario, ¿no estamos otorgando con estas cuestiones relaciones de la esfera de la ética, la moral, la virtud, las cuales han sido y son exigibles y adjudicables a los sujetos del Derecho por el carácter intrínsecamente subjetivo de tal materia? No se puede exigir a una silla que se porte bien, sin 
embargo, a una persona física sí y, por otro lado, la exigencia a una persona jurídica reside en el requerimiento indirecto a las personas naturales que conforman la sociedad (incluso, si la participación se divide a su vez en otras empresas, pues, al final de la cadena habrá detrás como mínimo una persona a cargo y quien tomará los procesos deliberativos). Cuando se exige un buen comportamiento a una empresa, en verdad se exige a las personas que la conforman. El telos de las exigencias éticas y morales atañen al comportamiento de los sujetos, no de los objetos, pues en tal caso son las personas responsables de la cosa. En caso contrario, estaríamos ante una exigencia de ética de bienes muebles, como si un bien mueble pudiera cumplir con un código de conducta y ejemplificar un comportamiento moral por sí solo.

Por tanto, si a una silla no le exigimos que se porte bien; si tampoco se podría presentar o ser propuesta para unas elecciones y ostentar un derecho de sufragio activo o tener a su cargo empleados o, en definitiva, los que atañe a los derechos fundamentales, ¿por qué una IA o un robot inteligente sí iba a poder hacerlo o, sobre todo, por qué el empeño de que sí pueda o deba? ¿En verdad, se puede considerar que una IA o robot inteligente ser autor de una obra artística, científica o literaria (art. 2 LPI y 10 LPI) y que goza de una "originalidad digital" (Lee, 2012), ¿y una "creatividad computacional" entendida según López de Mántaras como "el estudio del desarrollo de software que presenta un comportamiento que sería considerado creativo en seres humanos. Este software creativo puede usarse en tareas anónimas, como inventar teorías matemáticas, escribir poesía, pintar cuadros y componer música" (López de Mántaras, 2017)? ¿Y una "autoría algorítmica" (Vásquez Leal, 2020)? O, incluso, si debe ostentar una personalidad jurídica, ser "persona electrónica" que en palabras de Valente:

Personalidad electrónica significaría considerar a los robots como una persona de derecho que tiene ciertos derechos y obligaciones de carácter meramente instrumental para un interés económico específico de un ser humano. ${ }^{2}$

\footnotetext{
${ }^{2}$ Valente, L. A., 2019. La persona electrónica. Anales De La Facultad De Ciencias Juridicas
} Y Sociales De La Universidad Nacional De La Plat, Issue 49(01), p. 13. 
¿Acaso va a ostentar la IA derechos a la intimidad, honor y propia imagen?, ¿gozará en tal hipótesis de una dignidad digital? Todos estos escenarios cuando, en el mejor de los casos o el más revolucionario, se podría alcanzar, como mucho, un sujeto amoral, y en el suceso más plausible, se podría plantear una posición intermedia entre el objeto y el sujeto (Chávez Valdivia, 2020: 181-182), es decir, que se pueda escapar algo de la mera consideración de cosa o bien mueble por el hecho de que, a diferencia de la tan mencionada silla, una IA tiene la funcionalidad de actuar en campos que exigen un proceso deliberativo y decisorio, la función volitiva de tomar decisiones (probabilísticas en el fondo). Sin embargo, que una IA pueda desempeñar técnicas en disciplinas de la persona humana no implica que sea una persona (y mucho menos humana), pero no porque emane de la IA la reclamación de tal consideración, sino porque puede tener una utilidad práctica para resolver determinadas situaciones de gran complejidad. Que una IA pueda producir un cuadro, una canción, un poema no implica que tenga el animus vivendi, el incentivo creador intrínseco del ser humano, el cual no es programable, pues no puede ser "autora de algo que sólo puede hacer una persona física o natural" (Rodríguez Tapia, 2009). A nivel técnico puede ser una obra aceptable, incluso magnífica, a nivel técnico, pero ¿por qué no se protegería que una IA sobre un lienzo pintase un punto amarillo (ejemplo reduccionista de un cuadro abstracto) y si lo hace el ser humano sí?

La concepción de originalidad, creatividad, autoría o persona que se proyecta se legitima desde una definición negativa, pues es la no intervención humana lo que nos lleva a poder plantear que es la IA quien interviene de un modo "independiente", pero basta cualquier grado de intervención humana para entonces carecer de tal fundamento, al contario de un ser humano, cuya legitimación es afirmativa, parte del propio sujeto y aun con cualquier tipo o grado de intervención de un tercero, no pierde este ni un ápice de su titularidad ni originalidad, requisito de originalidad el cual, en palabras de Bercovitz Rodríguez-Cano, "es el requisito esencial para que una creación sea considerada obra” (Bercovitz Rodríguez-Cano, 1992). 
Sobre el sujeto entendido como aquel que pueda reclamarlo, según las premisas del norteamericano W. N. Hohfeld ${ }^{3}$, pone en relieve Sauca en las modalidades del derecho subjetivo (lo referido a pretensión, libertad, potestad e inmunidad, categorías de W. N. Hohfeld) en cuanto a la potestad, que "se encuentra en la situación jurídica de potestad aquel que dispone de un tipo de derecho subjetivo tal que puede modificar sus relaciones jurídicas frente a otro" (Sauca, 2000). Y en tal asunto, una IA o un robot inteligente no son capaces de modificar las relaciones jurídicas frente a otros individuos terceros, sí puede interaccionar, al igual que una sentencia, un acto administrativo, un contrato interacciona y no por ello nos apresuramos a darle una personalidad jurídica para que sean sujetos de Derecho, a menos que se quiera sobrepasar la ficción y concebir a un contrato como puede ser una compra de supermercado como un sujeto propio de Derecho.

En muchos casos, sobrepasando el campo de la realidad a través de la ficción mediante una realidad teórica, una realidad hipotética, dentro de la Filosofía del Derecho, y del Derecho en general, se ha sobrepasado la fictio legis que inspira toda norma y que es el telón de fondo entra la aparente neutralidad o imparcialidad de la norma y el factor axiológico que se oculta tras de sí. ¿Puede una IA o un robot reclamar derechos?, ¿pude llegar a ser un sujeto de Derecho a través de la pretensión o acción de reclamarlos? ¿No somos nosotros, en verdad, quienes proyectamos tales aspiraciones? ¿No reside el problema en que dentro de la personalidad que puedan sostener no es más que otro artificio regulatorio, una invención jurídica que se extrapola a de esa realidad formal a la realidad social?

El ejemplo más inminente es el de las personas jurídicas. Una empresa, por mucho empeño e ingeniería jurídica y filosófica que se proyecte, no escapa de ser una argucia como instrumento vehicular que sirva al ser humano en sus relaciones contractuales. Una empresa existe en sus estatutos, en los miembros societarios que lo conforman, sin embargo, nadie se puede tomar un café con bártulo de papeleo estatutario como tampoco se puede con la conceptualización abstracta de la empresa: necesitas que sea una persona, de carne y hueso,

${ }^{3}$ Véase Hohfeld, W. N., 1913. Conceptos jurídicos fundamentales. México: Fontamara, pp. 4587. 
con quien acuerdes tal citación, con quien pidas tal refrigerio descafeinado o no, con leche fría o caliente. Análogamente, con la IA, que es y no dejar de ser un bien mueble, por mucha pirueta lógica que se tercie, donde las exigencias de una ética, principios, valores, comportamiento o, por el contrario, la proyección de una bien mueble como sujeto capaz de reclamar derecho no son factibles, al igual que ninguna "empresa" como ente abstracto se presentó para reclamar derecho alguno, sino la persona (humana) que lo representaba sí lo hizo.

Entonces, en cuanto a la persona electrónica (propuesta que se formuló desde el Parlamento Europeo y la cual no estuvo exenta de controversias), sería crear una modalidad más a la de persona física y persona jurídica. En cambio, el principal problema de estas propuestas es que, si bien tras la persona jurídica habrá personas físicas que aporten el sujeto moral de la sociedad o empresa, en cambio, en la IA se propone sin un sujeto humano detrás, pues entonces no estaría este debate en cuestión (sería ese individuo el responsable y sujeto de Derecho, tal y como se ha venido haciendo hasta la actualidad): desde las propuestas de dotar de personalidad jurídica a las IAs y los robots inteligentes se propone con la capacidad tanto económica como moral del sujeto de Derecho a un ente que, en el mejor de los casos, no consigue llegar a ser más que un ente amoral, es más, es justo esta amoralidad intrínseca a la IA al aplicarse en campos éticos donde deliberaba el ser humano que se han producido los problemas, ya que el ser humano no es un ordenador ni un algoritmo es una persona (Copeland, 1993). Se pone un ente que no comprende qué es el bien ni el mal (dentro de una moral social consensuada y conforme a Derecho) a decidir como si estuviese en igualdad de condiciones de hacerlo que un ser humano.

A diferencia de las propuestas de una personalidad electrónica, sería mejor comprender una categoría intermedia entre objeto y sujeto, donde se acepta que es cierto que una IA como actúa en campos que antes podían ser exclusivos del ser humano (una silla no tenía la función deliberativa de determinar si se acepta la prestación económica a un ciudadano o no y una IA sí), no deja en esencia de ser un bien mueble capaz dentro del corpus mechanicum, es decir, dentro del ámbito técnico de aquellos procesos deliberativos-resolutivos donde participa, interactúa de un modo algo comparable a como haría una 
persona y, por tanto, trasciende en un pequeño grado a la situación de la cosa o bien como objeto de Derecho, pero que al estar exento de todo corpus mysticum, de toda voluntad incentiva (distinta a la iniciativa, que es programable) $\mathrm{y}$ de todo anymus vivendi, no alcanza a ser un sujeto pleno de Derecho, pues no escapará de ser un sujeto amoral. Y, por tanto, lo que precisa no es encuadrarlo en nuestros mismos parámetros, sino un derecho sui generis ${ }^{4}$, si acaso.

Entonces, esa clasificación intermedia comprende que, en el más avanzado de los casos, que se podría denominar personalidad ciberhumanoide sería entre un supra objeto y un cuasi sujeto, es decir, un sujeto limitado sólo a los ojos del Derecho (que no a la vista de la realidad social) para ser un sujeto económico con que responder de manera civil por los daños causados $\mathrm{y}$, por ende, para poder responder a tales demandas, la capacidad de custodiar un patrimonio dinerario que satisfaga así las pretensiones del tercero, no como el sujeto moral que sí es una persona física o una persona jurídica de manera indirecta por los individuos que la componen, ¿por qué? Pues debido a la ausencia humana en los procesos deliberativos en los cuales se implica a la IA. $\mathrm{Al}$ igual que no sería justo por el lejano o casi inexistente nexo causal entre ese proceso incognoscible para el programador que es la "caja negra" hacerle responsable de las decisiones que ha tomado una IA, por ejemplo, con sesgos racistas o de otra índole discriminatoria. Programador el cual puede respetar todos los derechos civiles de todo individuo, incluso ser el mayor defensor o hasta constar de tales características identitarias y el cual ni programó ni pudo prevenir ni sabe ni lo más mínimo en la toma de tales decisiones (esto se extiende al usuario de la IA), resultaría injusto adjudicarle la responsabilidad de tales actos. Por el contrario, es de suponer análogamente igual de injusto que los efectos de una acción sobre un tercero resulten exentos de su responsabilidad.

Con ello, ante la ausencia por parte del factor humano imputable de la dañosidad del acto y ante la prudencia de enquistar en una vacatio legis, se puede concebir desde una proyección jurídica el escenario de esa categoría intermedia, sin embargo, no se debería extrapolar esta situación desde una perspectiva

${ }^{4}$ Véase Ríos Ruiz, W. R., 2001. Los sistemas de inteligencia artificial y la propiedad intelectual de las obras creadas, producidas o generadas mediante ordenador. La Propiedad inmaterial, 13, p. 11 
antropológica a la realidad social, pues sería confundir el principio de $u b i$ lex non distinguit, nec nos distinguere debemus al factor humano, es decir, si por un hecho podemos no distinguir a quien lo causa, razonar que los posibles causantes son análogos o semejables. Y esto es lo que sucede, por ejemplo, con la creación y la autoría. En otras palabras, aunque en el ámbito jurídico, por el artificio mismo que lo define, sería factible como instrumento vehicular una personalidad intermedia como la propuesta, no escapa de ser una argucia jurídica, sin olvidar que sería para las excepcionales situaciones de las IAs más avanzadas.

\section{4. ¿Un objeto autor?:¿es una IA autor de una obra por el hecho de poder originarla?}

La propuesta de considerar una IA como autor supone aceptar un tipo de proceso donde un objeto puede "evolucionar" a sujeto, ya que no se debe descuidar el hecho de que por muy avanzada que sea o llegue a ser la IA, su código fuente, los algoritmos que la integren, son protegidos como objeto a través de la Propiedad Intelectual como obra, ya que su naturaleza es análoga a la de cualquier otra variedad de creación protegible. Es, en esencia, objeto, porque así es creado, a diferencia de una persona física, que nace como sujeto y se le reconoce su personalidad desde el nacimiento (incluso, la protección de sus derechos e interés antes en la figura del non nato) y a la persona jurídica que nace en el momento en el cual se formaliza conforme a la Ley y sus requisitos: ambos casos, desde su primer instante, nacen como sujetos de Derecho y no hay modo alguno que se cerciore ni vulnere tal condición, en cambio, la IA nace y se confiere desde su origen como objeto e, incluso, cuando "crea" una obra, por muy independiente y particular que sea tal hecho, lo hace desde su condición jurídica de objeto, de obra, de bien mueble.

Entonces, a través de una lectura inductiva, considerar que una IA desde criterios meritorios por el resultado, como una especie de iuris tantum, autor o con derechos de autor supone una ubicuidad normativa del mismo bien jurídico. La IA está registrada y se protege como objeto, pero sus resultados u 
obras se protegen con la IA como sujeto. ¿Cómo lidiaría el Derecho y la Filosofía con un ente afecto a esta duplicidad? Como el gato de Schrödinger, así la IA estaría siendo objeto y sujeto al mismo tiempo en dos planos jurídicos distintos.

Esto supone el plantear que una IA puede ostentar derechos ya no solo subjetivos sino fundamentales, pues en verdad resulta la libertad de creación un subgénero de la libertad de expresión. Entre los requisitos más esenciales para ser autor o inventor (dependiendo de si, en el primer caso, estamos en la Propiedad Intelectual o, en el segundo, en la Propiedad Industrial, aunque desde la OMPI y la mayoría de regulaciones anglosajonas el Property law aúna las dos disciplinas), destaca desde una visión subjetiva el factor del ingenio humano, la inventiva humana y la personalidad humana que se refleja sobre la obra o creación y, desde un ámbito objetivo, la necesidad de gozar de personalidad jurídica para poder ostentar o la titularidad directa de los derechos, es decir, el autor jurídico o, en todo caso, ser autor material.

Es más, de esta distinción objetiva inspirada en Locke sobre su concepción de la propiedad y de la teoría de los frutos se empezó a permitir el artificio jurídico de ser una persona jurídica el titular de derechos como empleador y el autor material el empleado que crea la obra (es la figura del Work Made For Hire). O, desde la segunda visión utilitarista de los planteamientos de Locke en este campo en el principio de la eficiencia y el "mejor uso", sobre los cuales se sostiene que reconocer a una IA o un robot inteligente de Derechos subjetivos y morales como son los de autor resulta eficiente y práctico, no sólo para el Derecho, sino para la realidad social, supone desconceptualizar o "vulgarizar" las cualidades a los conceptos de originalidad, creatividad y autoría de sus elementos más vitales, pues son características intrínsecas al corpus mysticum de lo que se protege y lo que representa la obra, que es la personalidad del autor, la huella, el ingenio humano que es elemento distinto al corpus mechanicum técnico de la obra. Con anterioridad se mencionó el ejemplo del arte abstracto, el cual se centra en esa cualidad subjetiva, la personalidad, y si frente un cuadro abstracto que sí sería protegible y se comprendería su protección, esto sería impensable si ese mismo cuadro lo ejecutara una IA, porque el motivo por el cual se reflexiona sobre la obra hecha por IA es, en verdad, por el factor meritorio de que la obra resultante a nivel técnico es semejable a 
la humana, es decir, que se considera que el resultado puede ser "original" porque emula a nivel técnico y meritorio un resultado que podría haber generado una persona. Por eso, alarma cuando se encuentra que en la sentencia china del Tribunal de Shenzen, de la provincia de Guandong, se protege con el mismo copyright que ostenta un autor humano a una obra producida por una IA al encontrar una estructura lógica, coherente y un cierto grado de originalidad (Fernández Carballo-Calero, 2021).

¿A qué originalidad se refiere? Pues no se cuestiona que una obra deba ser producida ex nihilo, ni tampoco se alude a que la creación debe ser ex novo, ya que cualquier autor o inventor se nutre de toda la herencia cultural e intelectual para formar su propia personalidad creativa (Antequera Parilli, 2007) y así crear con la aportación insoslayable de su personalidad e ingenio humano y a través de la técnica. Sin embargo, de los dos elementos indispensables del autor, por encontrar que una obra producida por IA puede cumplir con uno de ellos (que a nivel técnico sea en cierto grado semejable), no se debe apresurar mediante una lectura retrospectiva a concluir que cumple con la suma de todos los requisitos, pues el anymus, el incentivo creativo no existe en la IA, es en todo caso el mero espejismo ensayístico-probabilístico de un combate algoritmo dentro de su proceso de input y output.

Con ello, se puede en colación al apartado anterior, aplicando la creación instrumental de la personalidad intermedia, comprender que en el mejor de los casos se está ante una cuasi autoría como autoría limitada o secundaria donde se puede aceptar que el corpus mechanicum como uno de los requisitos para la autoría lo cumple, en el sentido de que el resultado, a nivel técnico, es protegible por el interés jurídico que atañe, aunque carece de todo anymus pues al ser en el mejor y más ficticio de los casos un sujeto amoral, ni puede tener ni tiene un corpus mysticum ni un factor moral ni una personalidad que impregne la obra. Por tanto, siendo como mucho en esa cuasi autoría que se propone el autor material y técnico de la obra, no es un autor moral ni puede serlo del resultado. Al igual que poco espíritu se cabe esperar que nazca de las cuatro patas de una silla, poco espíritu creativo se puede esperar que nazca de la combinación frenética de algoritmos y códigos fuentes, de unos y ceros.

Además, porque de entre los planteamientos filosóficos-jurídicos plausibles para las opciones de ser una IA sujeto pleno, autor pleno, se fundamenta 
en un utilitarismo deshumanizado ya que se faculta en una eficiencia la cual se contradice en sí misma, pues si el "individuo" al que le estás reconociendo unos derechos subjetivos es un ente incapaz de reclamar tales derechos o incluso valorar un interés e incentivo en su reivindicación ya que es un sujeto amoral es, en verdad, más ineficiente dotarle de esa capacidad, donde se deber tener bien en cuenta que, al hacerlo, tal derecho se convierte en un ius prohibendi frente a terceros, ese ente que es un sujeto amoral ostentando derechos morales, es en sí incapaz en su totalidad de reclamarlos, por tanto, si cabe la posibilidad de separar y desgajar esa capacidad para que bien por representación legal, bien por como se adopte la figura del tercero (aunque una tutela o curatela "algorítmicas" recaen en el mismo asunto, ¿cuál es el interés moral de una IA?), esta posibilidad se desvanece, desvirtuada en un hipotético sujeto que ostenta un derecho que en ius prohibendi es el solo capacitado para ejercerlo resulta que no puede, frente a que un tercero sí hubiera podido. En resumen, como bien dice Saiz García, por mucho empeño que se lleve a cabo, "las máquinas no tienen, a diferencia del ser humano (al menos todavía y casi todos), conciencia ni afecto que puedan verse influidos por la protección exclusiva de su esfuerzo" (Saiz García, 2019). Con lo cual, las protecciones morales por las cuales emergen este tipo de derechos y que se sustentan en ese sentido exclusivo del esfuerzo de nuestra conciencia y sensibilidad carecerían de todo sentido axiológico con la partura a entes que solo cumplen, en el mejor de los casos, el requisito técnico, el corpus mechanicum, exlcuyendo no sólo un elemento esencial, sino quizás el más fundamental de ambos, el anymus del sujeto, el corpus mysticum de la obra, uno de las características diferenciadoras e indisolubles del ser humano, del sujeto pleno de Derecho.

\section{Conclusiones: ¿objeto y sujeto? La caja destapada}

En conclusión, a tenor de todo lo expuesto en este trabajo, se puede concluir en primer lugar que por amplia que sea la lluvia de sucesos anecdóticos y contradictorios que se suceden en la realidad, la Filosofía del Derecho no debe dejarse obnubilar por los fuegos de artificio y confundirlos con el fuego proteico por el cual emana. Desde la naturaleza del propio hecho que supone una IA, que es la de un objeto como obra de un individuo, el programador, 
que protegerá tal obra conforme, este sí, a sus derechos de autor, desvirtualizar por el sentido de la materia para "ascenderlo" a la categoría de sujeto de Derecho en un nivel, además, que fuese equiparable al del ser humano, ya sea como persona física, ya sea como integrante de la persona jurídica, significa confundir o reducir la complejidad y diversidad terminológica de demasiados conceptos, los cuales no son ya sencillos de por sí. Sin embargo, aunque bien es cierto que la IA tiene la capacidad de ser parte en procesos deliberativos que normalmente dependían del factor humano, esto no significa que lo conviertan en humano, sino que una de las propiedades elaboradas en ese objeto es la de poder llevar a cabo tales funciones.

En caso contrario, confundir el telos de un objeto de este modo, es concebir que una máquina capaz de realizar operaciones quirúrgicas debe ser considerada, por ende, cirujano; que un sistema de IA para datos predictivos dentro de la administración pública deber ser entendida como un funcionario público; que, en nuestro caso, un algoritmo, IA o robot inteligente, por la capacidad de producir una obra debe ostentar un derecho, por ende, de autor, cuando además está ya protegido como obra del programador que la elaboró. En esta línea, sin ínfimos los casos y muy restringido a un tipo en concreto la IA que puede ser capaz de desarrollar tal tipo de facultades, sin embargo, aun en el mejor de los casos, se ha podido esclarecer que no en la situación más avanzada e hipotética no trasciende más allá de un sujeto amoral en el mejor de los resultados, pues asignarle una aptitud ética o moral es aceptar que sea pueda dar una ética de bienes muebles, que los bienes muebles pueden cumplir una actitud o un comportamiento ético o, aún más, que un objeto tiene moral propia, algo imposible más allá del campo de la mitología y la literatura.

Por tanto, como en el experimento teórico de Schrödinger, el gato se encuentra dentro de la caja con un cuenco de veneno y hasta que no se destape, el gato está vivo y muerto a la vez. Si se destapa, en este caso, la "caja negra" del proceso algorítmico y descubrir si es objeto y sujeto, surge la realidad de su naturaleza (y no sólo jurídica): es un objeto. No un objeto-autor. No un objeto-sujeto. Un objeto-objeto que es regulado y protegible como obra conforme al derecho de autor, derecho que ostenta, precisamente, su programador, quien sí es sujeto. 


\section{Bibliografía}

Amado Osorio, N. E., (2020): El derecho de autor en la inteligencia artificial de machine learning. La propiedad inmaterial, Volumen 30, pp. 327-353.

Antequera Parilli, R., (2007): Estudios de derecho de autor y derecho afines. Madrid: Editorial Reus.

Azuaje Pirela, M. \& Finol González, D., (2020): Transparencia algorítmica y la Propiedad Intelectual y Industrial: tensiones y soluciones. Revista Propiedad Inmaterial, Issue 30, pp. 111-146.

Bendito Cañizares, M. T., (2021): Estadio intermedio de reflexión para una futura regulación de la ética en el espacio digital europeo: los principios de transparencia y accountability. Revista Aranzadi de Derecho y Nuevas Tencologías, Issue 5, pp. 1-57.

Bercovitz Rodríguez-Cano, R., (1992): Comentario a la sentencia del Tribunal Supremo, de 26 de octubre de 1992. Cuaderno Cívitas de Jurisprudencia Civil, Issue 30, pp. 959-970.

Chávez Valdivia, A., (2020): Rediseñando la titularidad de las obras: Inteligencia artificial y robótica. Revista chilena de Derecho y Tecnología, 9(2), pp. 153-185.

Copeland, J., (1993): Cap. 9 “are we computers?”. En: Artificial Intelligence: Philosophical Introduction. New Jersey: Wiley-Blackwell, pp. 180-204.

Corvalán, J. G., (2017): La primera inteligencia artificial predictiva al servicio de la Justicia: Prometea. $L A L E Y$, pp. 2-5.

Díaz-Limón, J. A., (2016): Daddy's Car: la inteligencia artificial como herramienta facilitadora de derechos de autor. La Propiedad Inmaterial, Issue 22, pp. 83-100.

Fernández Carballo-Calero, P., (2021): La Propiedad Intelectual de las obras creadas por Inteligencia Artificial. Navarra: Editorial Aranzadi.

Grandhi, N. M., (2020): ¿Puede la Inteligencia Artificial ser un sujeto de Derecho?. Simposio Argentino de Informática y Derecho (Jaiio), pp. 54-61.

Hohfeld, W. N., (1913): Conceptos jurídicos fundamentales. México: Fontamara.

Lee, E., (2012): Digital originality. Vanderbilt J. of Ent. and Tech. Law, 14(4), pp. 919957.

López de Mántaras, R., (2017): La inteligencia artificial y las artes. Hacia una creatividad computacional. En: El próximo paso: la vida exponencial. Madrid: BBVA, pp. 99-123.

Marcos, A., (2015): La mejora (de la vida) humana: una reflexión antropológica y ética. Madrid, Cátedra de Bioética, UPCO, Madrid, p. 4.

Peinado Gracia, J. I., (2018): Lección 11. La propiedad intelectual: derechos de autor y derechos afines. En: Lecciones de Derecho Mercantil. Volumen I. Navarra: Thomson ReutersCivitas, pp. 211-236.

Ríos Ruiz, W. R., (2001): Los sistemas de inteligencia artificial y la propiedad intelectual de las obras creadas, producidas o generadas mediante ordenador. La Propiedad inmaterial, Issue 3, pp. 5-13. 
Rodríguez Tapia, J. M., (2009): Título II: Sujeto, objeto y contenido. Capítulo I: sujetos. En: Comentarios a la Ley de Propiedad Intelectual. Navarra: Thomson Reuters-Civitas, pp. $97-$ 119.

Saiz García, C., (2019): Las obras creadas por sistemas de inteligencia artificial y su protección por el derecho de autor. Indret: Revista para el análisis del Derecho, Volumen 1, pp. $1-45$.

Santos González, M. J., (2017): Regulación legal de la robótica y la inteligencia artifical: retos del futuro. Revista jurídica de la Universidad de León, pp. 25-50.

Sauca, J. M., (2000): Lección undécima: Los conceptos jurídicos fundamentales (II). En: Curso de Teoría del Derecho. Madrid: MARCIAL PONS, pp. 284-286.

Valente, L. A., (2019): La persona electrónica. Anales De La Facultad De Ciencias Juridicas Y Sociales De La Universidad Nacional De La Plat, Issue 49(01), pp. 1-30.

Vásquez Leal, L., (2020): ¿Autoría algorítmica? Consideraciones sobre la autoría de las obras generadas por inteligencia artificial. Revista Iberoamericana de la Propiedad Intelectual, Issue 13, pp. 207-233.

Yanisky-Ravid, S., (2017): Generating Rembrandt: Artificial Intelligence, Copyright, and Accountability in the $3^{\text {a }}$ Era: The Human-like Authors Are Already Here: A New Model. Mich, St. L. Rev. 659, Issue 4, pp. 659-726.

Jorge Villalobos jorgevillalobosportales@gmail.com 